\title{
Ellipsoidal mass models with varying axial ratios
}

\author{
D. K. Chakraborty ${ }^{\star}$
}

\author{
School of Studies in Physics, Pt. Ravishankar Shukla University, Raipur 492 010, India \\ e-mail: ircrsu@sancharnet.in
}

Received 16 March 2004 / Accepted 10 May 2004

\begin{abstract}
A family of triaxial mass models is described, for which the density is stratified on coaxial ellipsoids with varying axial ratios. The contours of constant projected density are almost perfect ellipses with ellpticity variations and isophote twists, but with no significant high-order residuals. These models are generalization of the triaxial models of Stark. An application to a real galaxy is presented.
\end{abstract}

Key words. galaxies: photometry - galaxies: structure - galaxies: general

\section{Introduction}

CCD photometry of elliptical galaxies shows that the isophotes are approximately elliptical, and ellipticity and position angle vary with radius. A triaxial mass model can produce variations in ellipticity and position angle in its projection. A spherical model $\rho(r)$, with density $\rho$ as a function of radius $r$, can be made triaxial by considering the expression for $\rho(r)$ and replacing $r^{2}$ by $m^{2}=x^{2}+y^{2} / p^{2}+z^{2} / q^{2}$, where $(x, y, z)$ are the usual Cartesian coordinates. The density is stratified on coaxial ellipsoids. It was shown analytically that in case the axial ratios $p$ and $q$ are constants, the projected density is stratified on similar and aligned ellipses (Stark 1977; Binney 1985). It was demonstrated by Madejsky \& Mollenhoff (1990) that models with equal density coaxial ellipsoidal shells with radially varying axial ratios can generate a variety of ellipticity and position angle profiles.

Triaxial mass models of an alternative form are $\rho(r, \theta, \phi)=$ $\mathrm{f}(r)-\mathrm{g}(r) Y_{2}^{0}(\theta)+\mathrm{h}(r) Y_{2}^{2}(\theta, \phi)$, where $\rho$ is density in the usual spherical polar coordinates $(r, \theta, \phi), \mathrm{f}(r)$ is a spherical mass distribution (which is usually a widely studied model), $\mathrm{g}(r)$ and $\mathrm{h}(r)$ are two suitably chosen radial functions, $Y_{2}^{0}=$ $\frac{3}{2} \cos ^{2} \theta-\frac{1}{2}$ and $Y_{2}^{2}=3 \sin ^{2} \theta \cos 2 \phi$ are the usual spherical harmonics. These models also exhibit ellipticity variations and isophote twists in their projections (de Zeeuw \& Carollo 1996; Chakraborty \& Thakur 2000). We shall refer to these models as fgh models.

The advantage of the fgh model is that the potential is known explicitly, and that the projected surface density can be calculated easily (and often, analytically). A disadvantage might be that the use of only the lower order spherical harmonics terms generally leads to models that become peanut-shaped for large values of the flattening. The addition of higher spherical harmonics terms leads to more nearly ellipsoidal shapes

^ Also a Senior Research Associate of IUCAA, Pune, India.
(Schwarzschild 1993), and "refines" the fgh models (de Zeeuw \& Carollo 1996). The isophotes of fgh models with higherorder spherical harmonics terms have significant high-order residuals (Chakraborty \& Das 2003).

We now present a family of mass models in which the surfaces of constatnt density are coaxial ellipsoids with varying axial ratios. The contours of constant projected density are almost perfect ellipses with varying ellipticity and position angle. High-order residuals are negligible. These features may be used to select a galaxy which may be suitable for comparison with the model. We apply our model to NGC 3379. This galaxy has almost perfectly elliptical isophotes (Lauer 1985) with a negligible isophote twist and a very little ellipticity variation (Peletier et al. 1990). The intrinsic shape of this galaxy has been determined by Statler (1994b). We use these results to gain some insight into the possible variation in the intrinsic shape of our model between its inner and outer regions, so that it may reproduce the photometric data of the galaxy to some extent. We find that the model which has a constant triaxiality parameter, and which is rounder in inner region and flatter in outer region, reproduces the data better.

In Sect. 2, we describe the mass model and in Sect. 3, we present the projected properties. Section 4 is devoted to a summary of the results and a discussion.

\section{Mass model}

We consider a density distribution of the form

$\rho=\rho\left(M^{2}\right)$

where

$M^{2}=x^{2}+\frac{y^{2}}{P^{2}}+\frac{z^{2}}{Q^{2}}$ 
with

$P^{-2}=\frac{b^{2 n} p_{0}^{-2}+b^{2 n-2} M^{2} p_{2}^{-2}+, \ldots,+M^{2 n} p_{2 n}^{-2}}{b^{2 n}+M^{2} b^{2 n-2}+, \ldots,+M^{2 n}}$

and a similar expression for $Q^{-2}$ in terms of $\left(q_{0}, q_{2}, \ldots, q_{2 n}\right)$. In the above, $\left(p_{2 i}, q_{2 i}\right),(i=0,1, \ldots, n)$ are parameters and $b$ is the scale length. We refer to Eq. $(3)$ as a $(n+1)$ point formula. It is clear that the constant density surfaces are coaxial and concentric ellipsoids, with the varying axial ratios $(P, Q)$ which are $\left(p_{0}, q_{0}\right)$ at small radii and $\left(p_{2 n}, q_{2 n}\right)$ at large radii. It is required that $0 \leq Q \leq P \leq 1$.

Substituting Eqs. (3) in (2), we obtain

$M^{2 n+2}+M^{2 n}\left(b^{2}-m_{2 n}^{2}\right)+, \ldots,-b^{2 n} m_{0}^{2}=0$,

where

$m_{2 i}^{2}=x^{2}+\frac{y^{2}}{p_{2 i}^{2}}+\frac{z^{2}}{q_{2 i}^{2}}$.

Equation (4) is used to calculate $M^{2}$ in terms of $x, y, z, p_{2 i}$ and $q_{2 i}$, which can be done numerically for a higher $n$. For a lower $n$, analytical methods can be applied. The model is fixed once the parameters $\left(p_{2 i}, q_{2 i}\right),(i=0,1, \ldots, n)$ are chosen, and $M^{2}$ is substituted in Eq. (1) with a chosen form of $\rho$. The scale length $b$ is calculated in terms of the effective radius $R_{\mathrm{e}}$. We shall refer to these models as $M^{2}$ models.

Presently, we confine ourselves to a 3-point formula $(n=2)$, so that Eq. (4) is sufficiently simple to solve, and still, variations in the axial ratios have a large variety of forms.

\subsection{3-point formula}

Rewriting Eq. (4) for $n=2$, we have

$M^{6}+M^{4}\left(b^{2}-m_{4}^{2}\right)+M^{2} b^{2}\left(b^{2}-m_{2}^{2}\right)-b^{4} m_{0}^{2}=0$,

which can be solved for $M^{2}$ analytically.

We consider two cases:

case (a) $p_{0}<p_{2}<p_{4}$ or $p_{0}>p_{2}>p_{4}$,

case (b) $p_{0}=p_{4}$ and $p_{2} \neq p_{0}$.

In each case, we take similar relations between $q_{2 i}$ 's, $(i=$ $0,1,2)$.

In case (a), $p_{2}$ is the axial ratio at a distance

$M_{p}^{4}=\frac{b^{4}\left(p_{2}^{-2}-p_{0}^{-2}\right)}{p_{4}^{-2}-p_{2}^{-2}}$

and $P$ increases (decreases) monotonically with $M$ when $p_{2 i}$ are in increasing (decreasing) order. In case (b), $P$ has the extremum value

$P_{\mathrm{E}}^{2}=\frac{3 p_{0}^{2} p_{2}^{2}}{2 p_{2}^{2}+p_{0}^{2}}$

at $M=b$. The extremum is the minimum (maximum) when $p_{2}$ is smaller (larger) than $p_{0}$. We have similar relations for $M_{q}^{4}$ and for $Q_{\mathrm{E}}^{2}$.

For a choice of $\left(p_{0}, p_{4}\right)$, the value of $p_{2}$ can be altered to vary $M_{p}$ for case (a), or $P_{\mathrm{E}}$ for case (b). Further, $P_{\mathrm{E}}$ can be larger or smaller than $p_{0}\left(=p_{4}\right)$ for case (b). These allow a greater flexibility in choosing the profiles of axial ratios in the $M^{2}$ models as compared to the fgh models. The latter model is fixed by assigning the values of axial ratios $\left(p_{0}, q_{0}\right)$ at small radii and $\left(p_{\infty}, q_{\infty}\right)$ at large radii. Choosing $p_{0}=p_{\infty}$ and $q_{0}=q_{\infty}$, similar to case (b) of the $M^{2}$ model, we find that axial ratios of the fgh model pass through minima at some intermediate radii (see, Fig. 1 of Chakraborty \& Das 2003).

We consider the form of $\rho$ as a generalization of Dehnen's (1993) $\gamma$ models, and take

$\rho(M)=\frac{(3-\gamma) M_{0} b}{4 \pi P Q} M^{-\gamma}(b+M)^{-4+\gamma}$,

where $M_{0}$ is the mass of the model, and $0 \leq \gamma<3$. The density decreases as $M^{-4}$ at large radii, and has a cusp rising as $M^{-\gamma}$ at small radii.

\section{Projected properties}

We follow the procedure laid by Binney (1985) very closely, to project $\rho$. Denoting the coordinates of the observer as $\left(x^{\prime}, y^{\prime}, z^{\prime}\right)$, with $z^{\prime}$ along the line of sight at the standard spherical polar cordinates $\left(\theta^{\prime}, \phi^{\prime}\right)$, and transforming the coordinates from $(x, y, z)$ to $\left(x^{\prime}, y^{\prime}, z^{\prime}\right)$, we write $m_{4}$ (here, we denote $p_{4}, q_{4}, m_{4}$ as $p, q, m$, respectively) as

$m^{2}=f z^{\prime 2}+g z^{\prime}+h$,

with $f, g, h$ the same as in Binney (1985). Introducing $z^{\prime \prime}$ by

$z^{\prime}=\frac{z^{\prime \prime}}{\sqrt{ } f}-\frac{g}{2 f}$

we write

$m^{2}=z^{\prime \prime 2}+h-\frac{g^{2}}{4 f} \equiv z^{\prime \prime 2}+a_{s}^{2}$.

Similar transformations of $m_{0}^{2}$ give

$m_{0}^{2}=f_{0} z^{2}+g_{0} z^{\prime}+h_{0}$,

where $f_{0}, g_{0}, h_{0}$ are the same as $f, g, h$ with $(p, q)$ replaced by $\left(p_{0}, q_{0}\right)$. Using Eqs. (11) in (13), we obtain

$m_{0}^{2}=P_{0} z^{\prime \prime 2}+R_{0} z^{\prime \prime}+Q_{0}^{2}$,

where

$P_{0}=\frac{f_{0}}{f}$,

$R_{0}=\frac{z^{\prime \prime}}{f \sqrt{ } f}\left(g_{0} f-g f_{0}\right)$

$Q_{0}^{2}=\frac{1}{4 f^{2} f_{0}}\left(g_{0} f-g f_{0}\right)^{2}+h_{0}-\frac{g_{0}^{2}}{4 f_{0}}$.

We find similar expressions for $m_{2}$ in terms of $f_{2}, g_{2}, h_{2}, P_{2}$, $R_{2}, Q_{2}^{2}$. In above, $a_{s}^{2}, Q_{0}^{2}, Q_{2}^{2}$ are function of $\left(x^{\prime 2}, x^{\prime} y^{\prime}, y^{\prime 2}\right)$, $R_{0}, R_{2}$ depend on $\left(x^{\prime}, y^{\prime}\right)$, and $f, f_{0}, f_{2}$ and $P_{0}, P_{2}$ are independent of the coordinates. We use relation (14) and a similar relation for $m_{2}^{2}$, along with relation (12) in Eq. (6), and solve for $M^{2}$. Substituting it in Eq. (9), we obtain $\rho$ as a function of $z^{\prime \prime}$. 
Table 1. The profiles of ellipticity $\epsilon$, position angle $\Theta_{\star}$ and surface density $\Sigma$, all as a function of the semi-major axis length $A$, expressed in terms of the scale length $b$, for case (a) of the $M^{2}$ model.

\begin{tabular}{lcccr|c}
\hline \hline$A / b$ & $\epsilon$ & $\Theta_{\star}$ & $\Sigma$ & rms deviation in $\Sigma$ & Comments \\
\hline 0.19 & 0.15 & 11.14 & 7.38 & $4703 \mathrm{e}-8$ & $p_{2 i}$ are $0.9,0.8,0.7$ \\
1.44 & 0.29 & 15.27 & 0.36 & $4807 \mathrm{e}-8$ & $q_{2 i}$ are $0.8,0.7,0.6$ \\
2.68 & 0.31 & 15.61 & 0.09 & $94 \mathrm{e}-8$ & $M_{p}^{2} / b^{2}=0.83$ \\
3.94 & 0.32 & 15.67 & 0.03 & $8 \mathrm{e}-8$ & $M_{q}^{2} / b^{2}=0.80$ \\
5.18 & 0.32 & 15.69 & 0.02 & $2 \mathrm{e}-8$ & $\theta^{\prime}=57.3^{\circ}$ \\
6.43 & 0.32 & 15.71 & 0.01 & $1 \mathrm{e}-8$ & $\phi^{\prime}=57.3^{\circ}$ \\
7.68 & 0.32 & 15.71 & 0.01 & $1 \mathrm{e}-8$ & \\
\hline
\end{tabular}

Table 2. The same as Table 1, for case (b) of the $M^{2}$ model.

\begin{tabular}{lcccr|c}
\hline \hline$A / b$ & $\epsilon$ & $\Theta_{\star}$ & $\Sigma$ & \multicolumn{1}{c}{ rms deviation in $\Sigma$} & \multicolumn{1}{c}{ Comments } \\
\hline 0.19 & 0.14 & 3.84 & 8.84 & $16102 \mathrm{e}-8$ & $p_{2 i}$ are $0.8,0.7,0.8$ \\
1.44 & 0.16 & 3.63 & 0.29 & $1450 \mathrm{e}-8$ & $q_{2 i}$ are $0.7,0.5,0.7$ \\
2.68 & 0.14 & 3.89 & 0.06 & $105 \mathrm{e}-8$ & $P_{\mathrm{E}}^{2}=0.76$ \\
3.94 & 0.13 & 4.02 & 0.02 & $12 \mathrm{e}-8$ & $Q_{\mathrm{E}}^{2}=0.61$ \\
5.18 & 0.13 & 4.07 & 0.01 & $3 \mathrm{e}-8$ & $\theta^{\prime}=80.0^{\circ}$ \\
6.43 & 0.12 & 4.11 & 0.01 & $1 \mathrm{e}-8$ & $\phi^{\prime}=20.0^{\circ}$ \\
7.68 & 0.12 & 4.13 & 0.00 & $0 \mathrm{e}-8$ & \\
\hline
\end{tabular}

The projected density $\Sigma\left(x^{\prime}, y^{\prime}\right)$ at a point $\left(x^{\prime}, y^{\prime}\right)$ in a plane perpendicular to the line of sight is

$\Sigma\left(x^{\prime}, y^{\prime}\right)=\int_{-\infty}^{\infty} \rho(M) \mathrm{d} z^{\prime}=\frac{1}{\sqrt{ } f} \int_{-\infty}^{\infty} \rho\left(z^{\prime \prime}\right) \mathrm{d} z^{\prime \prime}$.

We carry out the intregal (16) numerically. Defining the plane polar coordinates $\left.R=\sqrt{(} x^{\prime 2}+y^{\prime 2}\right)$ and $\Theta=\arctan \left(y^{\prime} / x^{\prime}\right)$, we evaluate Eq. (16) numerically on a circle of radius $R$. The angle at which $\Sigma(R, \Theta)$ is the maximum, gives position angle $\Theta_{\star}$ of the isophote with the semi-major axis length $A=R$. The semiminor axis length $B$ is determined by

$\Sigma\left(A, \Theta_{\star}\right)=\Sigma\left(B, \Theta_{\star}+\pi / 2\right)$.

\subsection{Isophotal characterstics}

We consider an ellipse with axes $A$ and $B$, and position angle $\Theta_{\star}$, and evaluate the projected density $\Sigma$ on the points of this ellipse. We find that the surface density is almost constant. The root mean square (rms) deviations in $\Sigma$ are extremely low, which in turn implies that the isophotes are almost perfect ellipses.

Tables 1 and 2 present the profiles of ellpticity $\epsilon=1-B / A$, position angle $\Theta_{\star}$ and the projected density $\Sigma$ for case (a) and for case (b), respectively, for the $M^{2}$ models. The rms deviations in $\Sigma$ are also shown. $A$ is the semi-major axis length, expressed in terms of the scale length $b$. The range of $A / b$ goes roughly from $0.15 R_{\mathrm{e}}$ to $6 R_{\mathrm{e}}\left(R_{\mathrm{e}}\right.$ is $1.28 b$ for the spherical $\gamma$ model, with $\gamma=1.5$ ). We take $\gamma=1.5$, for which the radial profile is closest to the de Vaucouleur's profile (Dehnen 1993). We also study the profiles of photometric parametres of the fgh models. We take the values of $\gamma$, viewing angles and the axial ratios at large and small radii the same as in case (b) of the $M^{2}$ models, so that the results can be compared.
We find that the rms deviationis in $\Sigma$ are extremely low in the $M^{2}$ models, even as compared to the fgh models. Deviation in $\Sigma$ in the $M^{2}$ models is particularly low in outer region, where we expect the axial ratios to be nearly constant at values $(p, q)$ throughout the line of sight, and therefore, the models should nearly reproduce Stark's result that the isophotes are perfect ellipses. We also show this analytically for a model with a 2-point formula (Appendix A). It was noted that the high order residuals are very low in the fgh models (see, e.g., de Zeeuw \& Carollo 1996). We conclude that the high order residuals are negligible in the $M^{2}$ models. We confirmed this seperately by finding $A_{4}, B_{4}$ coefficients. $B_{4}$ is typically $0.05 \%$ or less.

It is also seen from Table 1 that ellipticity changes monotonically, when $\left(p_{2 i}, q_{2 i}\right)$, and therefore, $(P, Q)$ change monotonically from inner region to outer region. This holds for case (a). For case (b), ellipticity passes through the extremum value at some intermediate $A$ (Table 2 ). The profiles of ellipticity of the fgh models exhibit variations similar to case (b).

Instead of considering $P$ and $Q$, we now consider $Q$ and triaxiality parameter $T$ defined by

$T=\frac{1-P^{2}}{1-Q^{2}}$

as intrinsic parameters. We find that position angle is almost constant when triaxality is constant, and ellipticity increases (decreases) monotonically when $Q$ decreases (increases) monotonically. This is a generalization of the results shown by Franx (1988) that the position angle of the Stark model depends on $T$ only, wrereas the ellipticity depends both on $T$ and $Q$. For this part of the study, we confine ourselves to case (a), and choose $q_{2 i}$ 's first and then calculate $p_{2 i}$ 's, so that

$t_{2 i}=\frac{1-p_{2 i}^{2}}{1-q_{2 i}^{2}}$ 
are same. The above choice makes $T$ practically constant. We exploit these results to gain some insight into the variation in the intrinsic shape of our model, so that the projected density may be compared with the photometric data of NGC 3379.

\subsection{Application to NGC 3379}

The intrinsic shape of NGC 3379 was determined by Statler (1994b). It is almost an oblate with the expectation values $\langle T\rangle=0.31,\langle Q\rangle=0.75$ and the most probable values $T_{\mathrm{P}}=0$, $Q_{\mathrm{P}}=0.87$, between $\sim 0.4 R_{\mathrm{e}}$ to $\sim 1.3 R_{\mathrm{e}}$. In this range, the position angle is practically constant and the ellipticity changes monotonically from $0.078 \pm 0.03$ to $0.133 \pm 0.02$ (Peletier et al. 1990). A comparison of these photometric data with the isophotal characterstics of our models suggests that we should consider a model wherein (i) triaxiality is almost constant, leading to a nearly constant position angle, and (ii) $Q$ decreases monotonically from inner to outer regions, leading to a monotonically increasing ellpticity. The $M^{2}$ models of case (a) with $q_{0}>q_{2}>q$ and constant triaxiality satisfy these requirements. Note that the $M^{2}$ models of case (b) and the fgh models are not satisfactory for this purpose, because axial ratios, and consequently, ellipticity do not exhibit monotonic variations.

In order to gain some quantitative estimate of the variation in the axial ratios of our model, we use the methodology described in Statler (1994a), based on Bayesian statistics. A summary of the methodology is presented in Thakur \& Chakraborty (2001). To calculate the probability of obtaining the observed data from a model, we consider a crude approximation of our model. We imagine that the model consists of three distinct regions: an outer region with the constant axial ratios $(p, q)$, an inner region with the constant axial ratios $\left(p_{0}, q_{0}\right)$ and an intermediate region. The ratios $(p, q)$ need not be the same as $\left(p_{0}, q_{0}\right)$. In intermediate region, axial ratios change from their values at outer region to those at inner region. The extent of these regions is not relevent, as long as they satisfy our assumptions, stated below, about the contributions to light along the line of sight, at a large and at a small radii.

When such a model is projected, let the line of sight at a large radius pierce through the outer region only. Hence, the model behaves as a Stark model, and we can use the formulas developed by Binney (1985), to calculate the ellipticity $\epsilon$, in terms of $\left(p, q, \theta^{\prime}, \phi^{\prime}\right)$. However, the line of sight at a small radius passes through all the three regions. The intermediate region is assumed to be very thin, and the contributions from outer region is treated as small compared to that from the inner region. As our model has a cusp at the center, this assumption is justified at small radii. The model can again be treated as Stark model, and the ellipticity $\epsilon_{0}$ can be calculated by Binney's formulas, in terms of $\left(p_{0}, q_{0}, \theta^{\prime}, \phi^{\prime}\right)$.

Considering the above crude model with a choice of $\left(q_{0}, q\right)$, we calculate $\left(p_{0}, p\right)$ and the predicted ellipticities $\epsilon_{0}$ and $\epsilon$, by taking $t_{0}=t=0.31,\left(t \equiv t_{4}\right)$. Using these and the corrosponding observed ellipticities, with errors $\sigma$ 's, we estimate the likelihood of obtaining the data from the model and the posterior density, by the formulas in Statler (1994a). We integrate the posterior density over the viewing angles $\left(\theta^{\prime}, \phi^{\prime}\right)$. The resultant

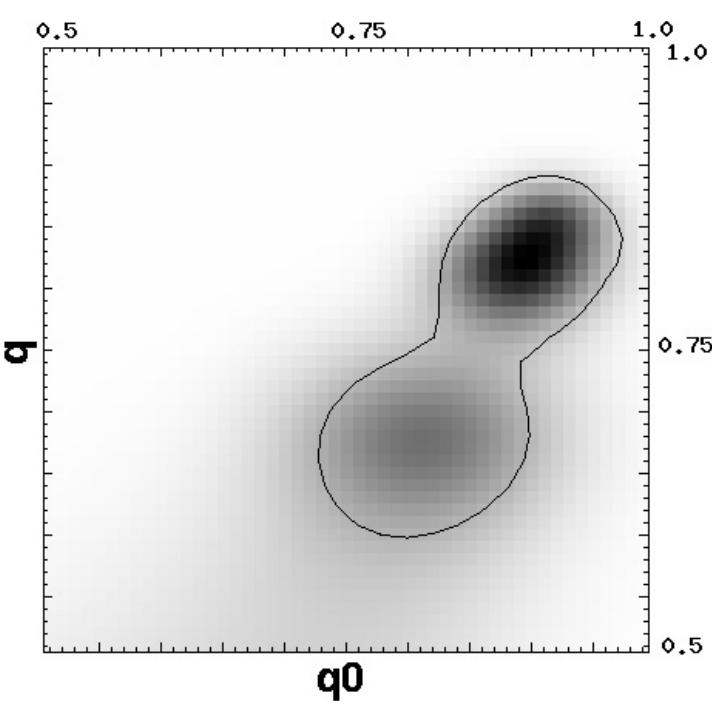

Fig. 1. Statistical estimate of the shape variation of the model, as a function of the intrinsic parameters $q_{0}, q$ and is shown in dark-grey shade. Darker is the shade, higher is the posterior density. The contour encloses the region of $\sim 1 \sigma$ error bar.

marginal posterior probability density $\mathcal{P}$ as a function of the shape parameters $\left(q_{0}, q\right)$ is presented in Fig. 1, and is shown by dark-grey shade. The darker is the shade, higher is the posterior density. The contour represents the $\sim 1 \sigma$ error bar region.

We find that the posterior density is sufficiently sharply peaked function of the shape parameters $q_{0}$ and $q$. This satisfies the requirement that the posterior density is relatively insensitive to the prior density. The higher values of $q$ and lower values of $q_{0}$ are in a region of very low probabilty. The most probable values are $q_{0 \mathrm{P}}=0.900, q_{\mathrm{P}}=0.830$ and the expected values are $\left\langle q_{0}\right\rangle=0.821,\langle q\rangle=0.703$.

In above, we have used the result $\langle T\rangle=0.31$. Now, we also use the result $\langle Q\rangle=0.75$ by applying condition $\frac{q_{0}+q}{2}=0.75$, and recalculate $\mathcal{P}$. We obtain $q_{\mathrm{OP}}=0.820, q_{\mathrm{P}}=0.680$, $\left\langle q_{0}\right\rangle=0.826,\langle q\rangle=0.674$. These results give the quantitative estimate of the shape variation of our model, so that it reproduces the data. Further, we integrate the likelihood over all $q_{0}$ and $q$, subjected to the condition $\frac{q_{0}+q}{2}=0.75$, and find the marginal likelihood distribution as a function of the viewing angles. The most probable viewing angles are $\theta^{\prime}=27^{\circ}, \phi^{\prime}=96^{\circ}$.

There is no specific reason to adopt $\langle T\rangle,\langle Q\rangle$ as the overall shape parameters of the galaxy. In fact, the posterior density is highly non-Gaussian, and therefore can not be characterized by only a few numbers. In addition to the expectation values, Statler (1994b) also gives the most probable values as $T_{\mathrm{P}}=0.0$, $Q_{\mathrm{P}}=0.87$. Calculating $\mathcal{P}$ by taking $t_{0}=t=0.0, \frac{q_{0}+q}{2}=0.87$, we obtain $q_{0 \mathrm{P}}=0.890, q_{\mathrm{p}}=0.850,\left\langle q_{0}\right\rangle=0.906,\langle q\rangle=0.834$. The most probable viewing angles for this shape is $\theta^{\prime}=117^{\circ}$, $\phi^{\prime}=54^{\circ}$.

In finding the above quantitive estimates, we have used the crude model, instead of our full $M^{2}$ model. The primary reason is the computation time. We now present the profiles of ellipticity for the $M^{2}$ models, with the choices of $q_{0}, q$, as calculated above, when the models are viewed along the most probable viewing angles. Value of $q_{2}$ is chosen as $\langle Q\rangle$ or $Q_{\mathrm{p}}$, as the case 
Table 3. The profiles of ellipticity $\epsilon^{1}$ and $\epsilon^{2}$ of the $M^{2}$ model. $\left(q_{0}, q_{2}, q, T, \theta^{\prime}, \phi^{\prime}\right)$ are $\left(0.826,0.750,0.674,0.31,27^{\circ}, 96^{\circ}\right)$ for $\epsilon^{1}$, and are $\left(0.906,0.870,0.834,0.0,117^{\circ}, 54^{\circ}\right)$ for $\epsilon^{2}$.

\begin{tabular}{cccc}
\hline \hline$A / b$ & $A / R_{\mathrm{e}}$ & $\epsilon^{1}$ & $\epsilon^{2}$ \\
\hline 0.128 & 0.1 & 0.076 & 0.075 \\
0.512 & 0.4 & 0.089 & 0.087 \\
0.896 & 0.7 & 0.106 & 0.104 \\
1.280 & 1.0 & 0.117 & 0.115 \\
1.664 & 1.3 & 0.123 & 0.121 \\
3.200 & 2.5 & 0.129 & 0.127 \\
4.352 & 3.4 & 0.130 & 0.128 \\
\hline
\end{tabular}

may be. Table 3 presents the profiles. We find the profiles of ellipticity are quite close to the observed profiles. There are disagreements regarding the radial distances at which the ellipticity is close to 0.078 or to 0.133 . This is due to the use of the crude model for the estimates of the shape variations.

\section{Results and discussion}

We have developed a mass model wherein the constant density surfaces are ellipsoids, with varying axial ratios. The contours of constant projected density are almost perfect ellipses, with ellipticity variations and posiation angle twists, and negligible high order residuals. In case, the triaxiality is nearly constant, the position angle is almost constant.

We have examined the variations in intrinsic shapes of our model, so that the photometric data of NGC 3379 may be reproduced. The model with constant triaxiality, which is rounder in inner region and flatter in outer region, is suitable for this purpose. A crude quantitative estimate of the change in axial ratios is made, which reproduces the data to some reasonable extent. Using a large ensemble of models, the methodology developed here may be applied to determine the shape variation of NGC 3379 itself.

Projection of the model has to be done numerically, and accuracy of a high order is required for the integration, so that the isophotal parameters may be calculated sufficiently accurately. Another disadvantage may be the calculation of the potential. This can be done, again numerically, using the multipole expansion (Binney \& Tremaine 1987).

Acknowledgements. D.K.C., Senior Research Associate of the InterUniversity Centre for Astronomy and Astrophysics (IUCAA), Pune, India expresses his sincere thanks to IUCAA for providing local hospitality and support during IUCAA visits. He also thanks Mr. M. K. Patil and Mr. S. Barway for discussions on IRAF packages. The author is grateful to the anonymous referee for his useful suggestions, which helped him to improve the paper.

\section{Appendix A}

For a 2-point formaula, Eq. (4) reduces to a quadratic formula in $M^{2}$ whose solutions are

$M^{2}=\frac{1}{2}\left\{\left(m_{2}^{2}-b^{2}\right) \pm\left[\left(m_{2}^{2}-b^{2}\right)^{2}+4 b^{2} m_{0}^{2}\right]^{\frac{1}{2}}\right\}$, and we retain only the postive sign to obtain positive $M^{2}$. We consider the triaxial modified Hubble model, with mass density

$\rho=\frac{\rho_{0} b^{3}}{\left(b^{2}+M^{2}\right)^{\frac{3}{2}}}$.

We take the difference between the axial ratios $\left(p_{0}, q_{0}\right)$ at small radii and $\left(p_{2}, q_{2}\right)$ at large radii to be small, and substituting Eqs. (A1) in (A2), we expand it in powers of the difference $\left(m_{2}^{2}-m_{0}^{2}\right)$. Retaining only the first order term, we obtain

$\rho=\rho_{0} b^{3}\left[\left(b^{2}+m_{2}^{2}\right)^{-\frac{3}{2}}+\frac{3}{2} \frac{b^{2}\left(m_{2}^{2}-m_{0}^{2}\right)}{\left(b^{2}+m_{2}^{2}\right)^{\frac{7}{2}}}\right]$.

Rewriting $p_{2}, q_{2}, m_{2}$ as $p, q, m$, we obtain relations (10) to (15). Putting these in Eq. (16), the projected density is

$$
\begin{aligned}
\Sigma= & \frac{2 \rho_{0} b^{3}}{\sqrt{ } f} \\
& \times\left\{\frac{1}{\left(b^{2}+a_{s}^{2}\right)}+\frac{b^{2}}{5}\left[\frac{1-P_{0}}{\left(b^{2}+a_{s}^{2}\right)^{2}}+\frac{4\left(a_{s}^{2}-Q_{0}^{2}\right)}{\left(b^{2}+a_{s}^{2}\right)^{3}}\right]\right\} .
\end{aligned}
$$

In Eq. (A4), the first term is the same as in Stark model, and the other terms are the contributions from the modifications introduced here. It is clear that at large distances the first term goes inversely as the square of the distance, whereas the other terms go inversely as the fourth power of the distance. Hence it fillows that at large distances, our model produces similar results as Stark model.

\section{References}

Binney, J. 1985, MNRAS, 212, 767

Binney, J., \& Tremaine S. 1987, Galactic dynamics (Princeton University Press)

Chakraborty, D. K., \& Das, M. 2003, A\&A, 402, 531

Chakraborty, D. K., \& Thakur, P. 2000, MNRAS, 318, 1273

de Zeeuw, P. T., \& Carollo, C. M. 1996, MNRAS, 281, 1333

Dehnen, W. 1993, MNRAS, 265, 250

Franx, M. 1988, MNRAS, 231, 285

Lauer, T. R. 1985, MNRAS, 216, 429

Madejsky, R., \& Mollenhoff, C. 1990, A\&A, 234, 119

Peletier, R. F., Davies, R. L., Illinworth, G. D., Davis, L. E., \& Cawson, M. 1990, AJ, 100, 1091

Schwarzschild, M. 1993, ApJ, 409, 563

Stark, A. A. 1977, ApJ, 213, 368

Statler, T. S. 1994a, ApJ, 425, 500

Statler, T. S. 1994b, AJ, 108, 111

Thakur, P., \& Chakraborty, D. K. 2001, MNRAS, 328, 330 